\title{
MELHORAMENTO GENÉTICO DE ERVA-MATE NATIVA DO ESTADO DE MATO GROSSO DO SUL $\left({ }^{1}\right)$
}

\author{
REGINALDO BRITO DA COSTA $\left({ }^{*}\right)$; MARCOS DEON VILELA DE RESENDE $\left({ }^{3}\right)$; \\ RAUL ALFFONSO RODRIGUES ROA $\left({ }^{4}\right)$; DAVI JOSÉ BUNGENSTAB $\left({ }^{5}\right)$; \\ WAGNER JOSÉ MARTINS $\left({ }^{6}\right)$; ANTONIA RAILDA ROEL $\left({ }^{7}\right)$
}

\begin{abstract}
RESUMO
O presente estudo objetivou avaliar geneticamente as melhores progênies de erva-mate, quanto aos caracteres de crescimento, provenientes de plantas nativas da área indígena de Caarapó, Mato Grosso do Sul. O teste de progênie foi instalado sob delineamento de blocos ao acaso com 30 tratamentos, 5 repetições e 10 plantas por parcela em linhas simples, no espaçamento $3 \times 2 \mathrm{~m}$. Aos 14 meses de idade, as progênies foram avaliadas quanto aos caracteres altura total das mudas e diâmetro do coleto. A herdabilidade individual no sentido restrito para os caracteres estudados pode ser considerada de média a alta magnitude $(0,15$ e 0,28), respectivamente, para altura e diâmetro. Os ganhos genéticos estimados com a seleção individual variaram para altura de 5,23 a $8,51 \mathrm{~cm}$ e para diâmetro de 2,08 a 3,15 $\mathrm{mm}$. Os valores genéticos preditos com propagação sexuada e assexuada indicam maiores possibilidades de ganhos com a implantação de plantios clonais. Na avaliação da sobreposição de geração, pelos resultados verifica-se que, em geral, os indivíduos da geração atual tendem a ser melhores do que aqueles provenientes da geração anterior.
\end{abstract}

Palavras-chave: Ilex paraguariensis, variabilidade genética, progênies, ganho genético.

\section{ABSTRACT \\ GENETIC IMPROVEMENT OF INDIGENOUS ERVA-MATE IN MATO GROSSO DO SUL STATE}

This study aimed at the genetic evaluation of growth of the best erva-mate progenies derived from native individual selected at the Indian reserve in Caarapó, Mato Grosso do Sul State. Experimental design of the progeny test was randomized blocks with 30 treatments, 5 replicates and 10 plants per plot in single rows at $3 \times 2 \mathrm{~m}$ spacing. Fourteen months old, progenies were evaluated for height and diameter of seedlings. Restrict individual heritability were of medium and high magnitudes $(0.15$ and 0.28$)$ for height and diameter respectively. Estimated genetic gains with individual selection ranged from 5.23 to $8.51 \mathrm{~cm}$ for height and from 2.08 to $3.15 \mathrm{~mm}$ for diameter. Predicted genetic values with sexual and asexual propagation indicate better gain potentials for the latter. Evaluation overlapping generations indicate that individuals from current generation tend to be better than the ones from the preceding generation.

Key words: Ilex paraguariensis, genetic variability, progenies, genetic gains.

( $\left.{ }^{1}\right)$ Recebido para publicação em 16 de setembro de 2008 e aceito em 19 de fevereiro de 2009.

$\left(^{2}\right)$ Universidade Federal de Mato Grosso (UFMT), Faculdade de Engenharia Florestal. Programa de Mestrado em Ciências Florestais e Ambiental, Av. Fernando Corrêa, s/n, 78060-900 Cuiabá (MT). E-mail: reg.brito.costa@gmail.com ( $\left.{ }^{*}\right)$ Autor correspondente. Bolsista do CNPq.

$\left({ }^{3}\right)$ Embrapa - Centro Nacional de Pesquisa de Florestas (CNPF), Caixa Postal 31, 83411-000 Colombo (PR). E-mail: marcos.deon@gmail.com

$\left({ }^{4}\right)$ Universidade Estadual Paulista Júlio de Mesquita Filho (UNESP), Programa de Pós-Graduação em Agronomia, Av. Brasil, 56, 15385-000 Ilha Solteira (SP). E-mail: roabio@gmail.com. Bolsista da CAPES.

$\left({ }^{5}\right)$ Universidade Estadual de Mato Grosso do Sul (UEMS), Av João Pedro Fernandes, 2101, Maracajú (MS). E-mail: bungenstab@bungenstab.com.br

$\left({ }^{6}\right)$ Universidade Federal de Mato Grosso do Sul (UFMS), Programa de Mestrado em Biologia Vegetal, Cidade Universitária, Caixa Postal 549, 79070-900, Campo Grande (MS). E-mail: martinswj@hotmail.com

$\left(^{7}\right)$ Universidade Católica Dom Bosco (UCDB), Programa de mestrado em Biotecnologia, Av. Tamandaré, 600, 79117-900 Campo Grande (MS). E-mail: ar_roel@hotmail.com 


\section{INTRODUÇÃO}

A erva-mate (Ilex paraguariensis St. Hil.), a espécie mais comum e utilizada do gênero, pertence à família Aquifoliaceae com cerca de 600 espécies. Destas, 220 são nativas da América do Sul e 68 ocorrem no Brasil (SCHERER, 1997; STURION e RESENDE, 1997). Pode-se considerar a erva-mate como dióica, em cujas plantas femininas se observam estames não funcionais e nas masculinas o pistilo se deprime e aborta (FERREIRA et al., 1983).

A cultura constitui uma das melhores opções de geração de emprego e de renda, especialmente entre os pequenos e médios produtores rurais. Ela forma um dos sistemas agroflorestais mais característicos da Região Sul do Brasil, sendo explorada em aproximadamente 600 empresas e 180 mil propriedades rurais, rendendo anualmente mais de R\$ 150 milhões (Rodigheri et al., 1995). É uma espécie perene bastante apreciada em todo o Brasil na forma de bebidas, insumos para alimentos, produtos de uso e higiene pessoal.

Depois do Uruguai, os maiores mercados em potencial ficam em países como Alemanha, Espanha, Itália, Estados Unidos (BoguszewsKI, 2007), Canadá e Japão (ANDrade, 1999), além do Oriente Médio, especialmente a Síria (Governo do PARANÁ, 2007). Este mercado continua em expansão, tanto na América do Sul como nos demais continentes, sendo a Argentina o maior produtor de mate no Cone Sul. No Brasil, a maior parte da produção é extrativista, demonstrando que a atividade ainda está muito dependente dos ervais nativos.

Por outro lado, o aumento da população e suas atividades, têm resultado na degradação e fragmentação de habitats causando declínio da biodiversidade (EHRLICH, 1988). A redução do tamanho original das áreas com cobertura vegetal nativa e o aumento do isolamento dos fragmentos florestais remanescentes têm provocado erosão genética em populações de I. paraguariensis, ao longo de anos de avanço da fronteira agrícola no Brasil, particularmente no Estado de Mato Grosso do Sul.

As populações nativas da espécie têm diminuído consistentemente o seu tamanho efetivo populacional levando à perda de variabilidade genética entre e dentro das populações. A detecção e resgate da referida variabilidade ainda presente nessas populações torna-se importante, a partir do conhecimento do povo Kaiowá e Guarani que mantiveram ao longo do tempo uma relação de tradição, rituais e consumo da espécie, com material genético ainda disponível em suas áreas que compreendem a divisa do Brasil e Paraguai.
A pesquisa de ANDRADE (1999) quantificou a produtividade média das árvores nativas, estabilizadas a partir dos 10 anos de idade, podendo chegar a produzir acima de $80 \mathrm{~kg}$ de matéria verde por árvore. Portanto, a disponibilidade de material genético autóctone para um mercado crescente de consumo nos diversos países citados deve fazer parte dos objetivos do melhoramento genético da erva-mate nativa do Brasil.

Nesse sentido, os testes de progênies, instrumentos importantes para o trabalho do melhorista, têm sido usados na estimativa de parâmetros genéticos e seleção de indivíduos, quando se procura avaliar a magnitude e a natureza da variância genética disponível, com vistas a quantificar e maximizar os ganhos genéticos, utilizando-se procedimento de seleção adequado.

O estudo objetivou avaliar geneticamente as melhores progênies de erva-mate, quanto aos caracteres de crescimento, provenientes de plantas nativas da área indígena de Caarapó, Mato Grosso do Sul.

\section{MATERIAL E MÉTODOS}

As sementes para a produção das mudas foram coletadas de matrizes nativas (polinização aberta), do município de Caarapó e em seu entorno, contando com a participação de membros da comunidade indígena Kaiowá e Guarani. Coletaramse amostras botânicas da espécie e as exsicatas foram depositadas no herbário da Universidade Federal de Mato Grosso do Sul (UFMS).

A Reserva Indígena Kaiowá e Guarani, cuja área total perfaz 3.600 hectares, está localizada na latitude $22^{\circ} 35^{\prime} \mathrm{S}$ e longitude $55^{\circ} 00^{\prime} \mathrm{W}$. O clima é de savana com duas estações bem definidas: uma quente e úmida e outra mais fria e seca com déficit hídrico, temperatura média anual de $20{ }^{\circ} \mathrm{C}$ e precipitações pluviais entre 1400 e $1700 \mathrm{~mm}$ bem distribuídas durante o ano.

O teste de progênie foi instalado sob delineamento de blocos ao acaso com 30 tratamentos (progênies), cinco repetições e 10 plantas por parcela em linhas simples, no espaçamento $3 \times 2 \mathrm{~m}$. Aos 14 meses de idade, as progênies foram avaliadas quanto aos caracteres: a) altura total das mudas expressas em centímetros e, b) diâmetro do coleto expresso em milímetros.

As variáveis foram analisadas usando-se a metodologia de modelo linear misto univariado aditivo do software SELEGEN - REML/BLUP (restricted maximum likelihood) apresentado por RESENDE (2002b), consistindo do seguinte: 


$$
\mathrm{y}=\mathrm{Xb}+\mathrm{Za}+\mathrm{Wc}+\mathrm{e}, \text { em que: }
$$

$\mathrm{y}, \mathrm{b}, \mathrm{a}, \mathrm{c}$, e: vetores de dados, dos efeitos das médias de blocos (aleatório), de efeitos genéticos aditivos (aleatório), de efeitos de parcela (aleatório) e de erros aleatórios, respectivamente.

$\mathrm{X}, \mathrm{Z}, \mathrm{W}$ : matrizes de incidência para $\mathrm{b}, \mathrm{a}, \mathrm{c}$, respectivamente. variâncias

Distribuições e estruturas de médias e

$$
\begin{aligned}
& y \mid b, V \sim N(X b, V) \\
& a \mid A, \hat{\sigma}_{a}^{2} \sim N\left(0, A \sigma_{a}^{2}\right) \\
& c \mid \hat{\sigma}_{c}^{2} \sim N\left(0, I \sigma_{c}^{2}\right) \\
& e \mid \hat{\sigma}_{e}^{2} \sim N\left(0, I \sigma_{e}^{2}\right) \\
& \operatorname{Cov}\left(a, c^{\prime}\right)=0 ; \operatorname{Cov}\left(a, e^{\prime}\right)=0 ; \operatorname{Cov}\left(c, e^{\prime}\right)=0 \\
& \text { ou seja: }
\end{aligned}
$$

$$
E\left[\begin{array}{c}
y \\
a \\
c \\
e
\end{array}\right]=\left[\begin{array}{c}
X b \\
0 \\
0 \\
0
\end{array}\right] \text { e Var }\left[\begin{array}{c}
y \\
a \\
c \\
e
\end{array}\right]=\left[\begin{array}{cccc}
V & Z G & W C & R \\
G Z^{\prime} & G & 0 & 0 \\
C W^{\prime} & 0 & C & 0 \\
R & 0 & 0 & R
\end{array}\right] \text {, em que: }
$$

$G=A \hat{\sigma}_{a}^{2}$

$R=I \hat{\sigma}_{c}^{2}$

$C=I \hat{\sigma}_{e}^{2}$

$V=Z A \hat{\sigma}_{a}^{2} Z^{\prime}+W I \hat{\sigma}_{c}^{2} W^{\prime}+I \hat{\sigma}_{e}^{2}=Z G Z^{\prime}+W C W^{\prime}+R$.

Equações de modelo misto

$$
\begin{aligned}
& {\left[\begin{array}{ccc}
X^{\prime} X & X^{\prime} Z & X^{\prime} W \\
Z^{\prime} X & Z^{\prime} Z+A^{-1} \lambda_{1} & Z^{\prime} W \\
W^{\prime} X & W^{\prime} Z & W^{\prime} W+I \lambda_{2}
\end{array}\right]\left[\begin{array}{l}
\hat{b} \\
\hat{a} \\
\hat{c}
\end{array}\right]=\left[\begin{array}{c}
X^{\prime} y \\
Z^{\prime} y \\
W^{\prime} y
\end{array}\right] \text {, em que: }} \\
& \lambda_{1}=\frac{\hat{\sigma}_{e}^{2}}{\hat{\sigma}_{a}^{2}}=\frac{1-\hat{h}^{2}-c^{2}}{\hat{h}^{2}} ; \quad \lambda_{2}=\frac{\hat{\sigma}_{e}^{2}}{\hat{\sigma}_{c}^{2}}=\frac{1-\hat{h}^{2}-\hat{c}^{2}}{\hat{c}^{2}} \\
& \hat{h}_{a}^{2}=\frac{\hat{\sigma}_{a}^{2}}{\hat{\sigma}_{a}^{2}+\hat{\sigma}_{c}^{2}+\hat{\sigma}_{e}^{2}}=\text { herdabilidade individual }
\end{aligned}
$$

no sentido restrito no bloco;

$$
\hat{h}_{m p}^{2}=\frac{0,25 \hat{\sigma}_{a}^{2}}{0,25 \hat{\sigma}_{a}^{2}+\hat{\sigma}_{c}^{2} / b+\hat{\sigma}_{e}^{2} /(n b)}=\text { herdabilidade }
$$

média de progênies no sentido restrito no bloco;

$$
\hat{c}^{2}=\hat{\sigma}_{c}^{2} /\left(\hat{\sigma}_{a}^{2}+\hat{\sigma}_{c}^{2}+\hat{\sigma}_{e}^{2}\right)=\text { correlação devida }
$$
ao ambiente comum da parcela;

$$
\begin{aligned}
& \hat{\sigma}_{a}^{2}=\text { variância genética aditiva; } \\
& \hat{\sigma}_{c}^{2}=\text { variância entre parcelas; }
\end{aligned}
$$

$$
\begin{aligned}
& \mathrm{CV}_{\mathrm{gi}}(\%)=\frac{\sqrt{\hat{\sigma}_{a}^{2}}}{\bar{X}} \cdot 100 \\
& \mathrm{CV}_{\mathrm{e}}(\%)=\frac{\sqrt{\hat{\sigma}_{e}^{2}}}{\bar{X}} \cdot 100 \\
& \hat{\sigma}_{e}^{2}=\text { variância residual dentro da parcela }
\end{aligned}
$$
(ambiental + não aditiva); individual;

$\mathrm{CV}_{\mathrm{gi}}=$ coeficiente de variação genética

$\mathrm{CV}_{\mathrm{e}}=$ coeficiente de variação experimental;

$\mathrm{A}=$ matriz de correlação genética aditiva entre os indivíduos em avaliação.

Acurácia seletiva $=$ obtida a partir da raiz quadrada da herdabilidade média de progênie.

A utilização da metodologia Reml/Blup, desenvolvida para o melhoramento de plantas perenes, tem maximizado os ganhos genéticos com seleção (RESENDE, 2002b, Costa et al., 2002; Costa et al., 2005; Missio et al., 2005), por se tratar de um procedimento estimativo, especialmente para dados desbalanceados, predizendo valores genéticos dos indivíduos em testes de progênies (RESENDE, 2002a).

\section{RESULTADOS E DISCUSSÃO}

Os resultados referentes às estimativas dos parâmetros genéticos para os caracteres altura, diâmetro e sobrevivência das progênies são apresentados na tabela 1 .

A herdabilidade individual no sentido restrito para os caracteres estudados podem ser consideradas de médias magnitudes $(0,15$ e 0,28$)$ para altura e diâmetro, respectivamente. Resultados mais expressivos foram anotados para média de progênies $(0,66$ e 0,78) para altura e diâmetro, respectivamente, revelado que a seleção pode ser efetiva usando-se as informações, tanto de famílias, quanto dos indivíduos. Esses valores são superiores àqueles observados por Costa et al. (2005) para progênies nativas da região, o que denota boa perspectiva de variabilidade genética a ser explorada ao longo de um programa de melhoramento genético. As informações de famílias são coerentes com os resultados verificados na literatura relativa a outras espécies florestais (KagEYAMA e VenCOVSKY, 1983; StURION, 1993; CoRNellius, 1994; SAMPAIO, 1996) e quanto à ervamate (SIMEÃO et al., 2002).

A mais importante função da herdabilidade no estudo genético do caráter métrico é seu papel preditivo expressando a confiança do valor fenotípico como um guia para o valor genético, ou o grau de correspondência entre o valor fenotípico e o valor genético (FAlConer, 1987; Vencovsky e BARRiga, 1992). 
Tabela 1. Estimativas de parâmetros genéticos para os caracteres altura e diâmetro em indivíduos de ervamate, aos 14 meses de idade, em Caarapó (MS)

\begin{tabular}{lcc}
\hline Estimativas $\left(^{1}\right)$ & Altura & Diâmetro \\
\hline$\hat{h}^{2}$ & $\mathrm{~cm}$ & $\mathrm{~mm}$ \\
$\hat{h}_{m p}^{2}$ & 0,1540 & 0,2810 \\
$\hat{\sigma}_{a}^{2}$ & 0,6633 & 0,7881 \\
$\hat{\sigma}_{c}^{2}$ & 32,8798 & 3,9655 \\
$\hat{\sigma}_{e}^{2}$ & 0,3727 & 0,0238 \\
$\hat{\sigma}_{f}^{2}$ & 180,1627 & 10,1220 \\
\hline Média geral $^{2}$ & 213,4152 & 14,1112 \\
$\mathrm{CV}_{\mathrm{gi}}(\%)$ & 32,5028 & 19,1805 \\
$\mathrm{CV}_{\mathrm{e}}(\%)$ & 17,6418 & 10,3823 \\
\hline
\end{tabular}

$\left({ }^{1}\right)$ Herdabilidade individual no sentido restrito no bloco $\left(\hat{h}^{2}\right)$; herdabilidade média de progênie $\left(\hat{h}_{m p}^{2}\right)$; variância genética aditiva $\left(\hat{\sigma}_{a}^{2}\right)$; variância ambiental entre parcelas $\left(\hat{\sigma}_{c}^{2}\right)$; variância residual dentro de parcela (ambiental + não aditiva, $\left.\hat{\sigma}_{e}^{2}\right)$; variância fenotípica individual $\left(\hat{\sigma}_{f}^{2}\right)$; coeficiente de variação genética individual $\left(\mathrm{CV}_{\mathrm{gi}} \%\right)$; coeficiente de variação experimental $\left(\mathrm{CV}_{\mathrm{e}} \%\right)$.

Segundo Falconer (1987), a herdabilidade é uma propriedade não somente de um caráter, mas também da população e das circunstâncias de ambientes às quais os indivíduos estão sujeitos.

Os coeficientes de variação genética individual $\left(\mathrm{CV}_{\mathrm{gi}} \%\right)$, que expressam em percentagem da média geral a quantidade de variação genética existente, revelaram valores expressivos para os caracteres em estudo $(17,64 \%$ e $10,38 \%)$ condizentes àqueles obtidos por Costa et al. (2005). Esses resultados revelam que a população pode ser considerada apropriada para o programa de melhoramento genético. Em outros termos, ganho genético é esperado aplicando-se procedimento adequado de seleção. Pelos dados obtidos sugere-se que, em futuras avaliações de campo, poderá haver maior expressão da variação genética para os caracteres estudados e produção de massa foliar associada.

Nos coeficientes de variação experimental $\left(\mathrm{CV}_{\mathrm{e}} \%\right)$, os valores de 6,02\% para diâmetro e $14,05 \%$ para altura foram considerados baixos e médios para os respectivos caracteres (GARCIA, 1989).

$\mathrm{Na}$ tabela 2 são apresentados os valores fenotípicos, genéticos aditivos dos dez melhores indivíduos, com base nos valores genéticos, ganhos genéticos preditos e nova média da população para o caráter altura.

Observa-se que os indivíduos das progênies 5, 20 e 21 tiveram melhores desempenhos, porém há uma preponderância sequencial dos indivíduos da progênie 20, que é promissora em relação aos valores genéticos. O valor expressivo de acurácia seletiva $(0,81)$ reforça a importância do uso da informação de progênie. Neste contexto, os valores preditos permitem indicar com mais segurança que o germoplasma em estudo maximizará as possibilidades de progresso genético com seleção. De maneira geral, os valores genéticos preditos não são iguais aos valores genéticos verdadeiros dos indivíduos.

Tabela 2. Valores fenotípicos, genéticos aditivos, ganhos genéticos preditos e nova média da população, dos 10 melhores indivíduos para o caráter altura $(\mathrm{cm})$ de erva-mate, aos 14 meses de idade, em Caarapó (MS)

\begin{tabular}{|c|c|c|c|c|c|c|c|}
\hline Ordem & Bloco & Progênie & Árvore & $\begin{array}{c}\text { Valores } \\
\text { fenotípicos }\end{array}$ & $\begin{array}{l}\text { Valores } \\
\text { genéticos }\end{array}$ & $\begin{array}{l}\text { Ganho } \\
\text { genético }\end{array}$ & $\begin{array}{l}\text { Nova } \\
\text { Média }\end{array}$ \\
\hline & & & & & $(u+a)$ & 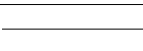 & 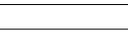 \\
\hline 1 & 4 & 5 & 1 & 96,5000 & 41,0180 & 8,5152 & 41,0180 \\
\hline 2 & 3 & 20 & 8 & 63,5000 & 38,6910 & 7,3517 & 39,8545 \\
\hline 3 & 2 & 20 & 7 & 58,7000 & 38,2182 & 6,8062 & 39,3091 \\
\hline 4 & 3 & 21 & 7 & 63,5000 & 37,3406 & 6,3141 & 38,8169 \\
\hline 5 & 2 & 20 & 8 & 50,8000 & 37,2688 & 6,0045 & 38,5073 \\
\hline 6 & 3 & 20 & 6 & 50,7000 & 37,1527 & 5,7787 & 38,2816 \\
\hline 7 & 1 & 16 & 9 & 53,5000 & 37,1406 & 5,6157 & 38,1186 \\
\hline 8 & 3 & 20 & 9 & 48,4000 & 36,8763 & 5,4605 & 37,9633 \\
\hline 9 & 2 & 9 & 10 & 59,3000 & 36,8566 & 5,3375 & 37,8403 \\
\hline 10 & 2 & 9 & 6 & 59,0000 & 36,6205 & 5,2355 & 37,7383 \\
\hline $\begin{array}{l}\text { Média anterior } \\
\text { da população }\end{array}$ & - & - & - & - & - & - & 32,50 \\
\hline Acurácia seletiva & - & - & - & - & - & - & 0,81 \\
\hline
\end{tabular}


A proximidade entre esses dois valores pode ser avaliada com base na estatística denominada acurácia (VAN VLECK et al., 1987). A literatura prática pertinente ao assunto tem demonstrado a importância da acurácia para apontar o grau de confiabilidade dos resultados na avaliação genética (RESENDE et al., 1995; Costa et al., 2000).

Os ganhos genéticos estimados com a seleção individual para o caráter altura elevaram a nova média da população após um ciclo de seleção de 32,50 $\mathrm{cm}$ para $41,02 \mathrm{~cm}$, equivalente a um percentual expressivo na média de $16,10 \%$.

Os valores fenotípicos, genéticos aditivos dos dez melhores indivíduos, ganhos genéticos preditos e nova média da população para o caráter diâmetro são apresentados na tabela 3 .

Em relação ao caráter diâmetro destacaram-se os indivíduos das progênies 16 e 22. De forma similar ao caráter altura, avaliações posteriores em idades mais avançadas poderão confirmar o desempenho desse material para efeito de seleção, visando maximizar o ganho genético. SIMEÃo et al. (2002) enfatizam que os valores genéticos preditos em relação a todos os indivíduos candidatos possibilitam estabelecer a melhor estratégia para o aumento da eficiência do melhoramento.

Os ganhos genéticos estimados com a seleção individual para o caráter diâmetro elevaram a nova média da população após um ciclo de seleção de 19,18 $\mathrm{mm}$ para $22,33 \mathrm{~mm}$, o equivalente a um percentual na média de $10,07 \%$.

$\mathrm{Na}$ tabela 4, relacionam-se os valores genéticos aditivos preditos $(\hat{\mu}+\hat{a})$ e genotípicos $(\hat{\mu}+\hat{g})$, assumindo-se grau médio de dominância igual a $1 \mathrm{em}$ uma população com nível intermediário de melhoramento, ganhos genéticos e nova média da população dos 10 melhores genótipos da erva-mate aos 14 meses de idade para o caráter altura.

Verifica-se que dentre o material selecionado, considerando-se propagação assexuada (ordenadas por $\hat{g}$ ) para altura (Tabela 4), o genótipo 20 foi comum à propagação sexuada (ordenadas por $\hat{a}$ ), porém a sequência dos melhores genótipos altera-se pelo tipo de propagação considerada. A seleção dos melhores indivíduos com base no sistema de propagação (assexuada ou sexuada) está intimamente ligada aos objetivos do programa de melhoramento da espécie. Se o objetivo visa à transformação do teste de progênie em pomar de semente por mudas, o ideal será selecionar os indivíduos com base em $\hat{a}$. Por outro lado, se o objetivo é fornecer material para instalação de um pomar de semente clonal, então deve-se selecionar os indivíduos com base em $\hat{g}$, para que haja maximização do ganho genético. Os valores genotípicos $(\hat{\mu}+\hat{g})$ preditos e genético aditivo $(\hat{\mu}+\hat{a})$ para os 10 melhores genótipos variaram de 38,40 a 45,65 e 36,82 a 41,02, respectivamente, para o caráter altura (Tabela 4 ).

Tabela 3. Valores fenotípicos, genéticos aditivos, ganhos genéticos preditos, número efetivo populacional e nova média da população, dos 10 melhores indivíduos para o caráter diâmetro $(\mathrm{mm})$ de erva-mate, aos 14 meses de idade, em Caarapó (MS)

\begin{tabular}{|c|c|c|c|c|c|c|c|}
\hline Ordem & Bloco & Progênie & Árvore & $\begin{array}{l}\text { Valores } \\
\text { fenotípicos }\end{array}$ & $\begin{array}{l}\text { Valores } \\
\text { genéticos }\end{array}$ & $\begin{array}{l}\text { Ganho } \\
\text { genético }\end{array}$ & $\begin{array}{l}\text { Nova } \\
\text { Média }\end{array}$ \\
\hline & & & & & $(u+a)$ & 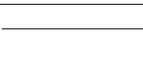 & \\
\hline 1 & 1 & 16 & 8 & 29,00 & 22,3334 & 3,1528 & 22,3334 \\
\hline 2 & 1 & 22 & 8 & 29,00 & 21,7576 & 2,8649 & 22,0455 \\
\hline 3 & 2 & 16 & 5 & 26,00 & 21,7272 & 2,7589 & 21,9394 \\
\hline 4 & 1 & 16 & 1 & 24,00 & 21,2000 & 2,5740 & 21,7545 \\
\hline 5 & 1 & 16 & 7 & 24,00 & 21,2000 & 2,4631 & 21,6436 \\
\hline 6 & 1 & 16 & 2 & 23,00 & 20,9733 & 2,3514 & 21,5319 \\
\hline 7 & 3 & 20 & 6 & 25,00 & 20,9451 & 2,2675 & 21,4480 \\
\hline 8 & 4 & 21 & 4 & 25,00 & 20,9419 & 2,2043 & 21,3848 \\
\hline 9 & 3 & 11 & 7 & 24,00 & 20,7928 & 2,1385 & 21,3190 \\
\hline 10 & 1 & 16 & 5 & 22,00 & 20,7466 & 2,0813 & 21,2618 \\
\hline $\begin{array}{l}\text { Média anterior } \\
\text { da população }\end{array}$ & - & - & - & - & - & - & 19,20 \\
\hline Acurácia seletiva & - & - & - & - & - & - & 0,89 \\
\hline
\end{tabular}


Tabela 4. Efeitos aditivos $(\hat{a})$, valores genéticos aditivos $(\hat{\mu}+\hat{a})$ preditos, efeitos genotípicos $(\hat{g})$ e valores genotípicos $(\hat{\mu}+\hat{g})$ para os 10 melhores genótipos de erva-mate aos 14 meses de idade, para o caráter altura, em Caarapó (MS)

\begin{tabular}{|c|c|c|c|c|c|c|c|c|c|}
\hline \multirow{2}{*}{ Bloco } & \multicolumn{2}{|c|}{ Propagação sexuada } & \multirow{2}{*}{$\hat{a}$} & \multirow{2}{*}{$\hat{\mu}+\hat{a}$} & \multirow{2}{*}{ Bloco } & \multicolumn{2}{|c|}{ Propagação assexuada } & \multirow{2}{*}{$\hat{g}$} & \multirow{2}{*}{$\hat{\mu}+\hat{g}$} \\
\hline & Genótipo & Indivíduo & & & & Genótipo & Indivíduo & & \\
\hline 4 & 5 & 1 & 8,52 & 41,02 & 4 & 5 & 1 & 13,14 & 45,65 \\
\hline 3 & 20 & 8 & 7,35 & 38,69 & 3 & 20 & 8 & 8,47 & 40,97 \\
\hline 2 & 20 & 7 & 6,81 & 38,22 & 2 & 20 & 7 & 7,69 & 40,19 \\
\hline 0 & 20 & 0 & 6,31 & 37,34 & 3 & 21 & 7 & 7,24 & 39,74 \\
\hline 3 & 21 & 7 & 3,00 & 37,27 & 2 & 9 & 10 & 6,49 & 39,00 \\
\hline 2 & 20 & 8 & 5,78 & 37,15 & 2 & 9 & 6 & 6,43 & 38,94 \\
\hline 3 & 20 & 6 & 5,62 & 37,14 & 1 & 16 & 9 & 6,32 & 38,82 \\
\hline 1 & 16 & 9 & 5,46 & 36,88 & 3 & 21 & 8 & 6,22 & 38,72 \\
\hline 3 & 20 & 9 & 5,34 & 36,86 & 2 & 20 & 8 & 6,09 & 38,60 \\
\hline 2 & 9 & 10 & 5,24 & 36,82 & 3 & 20 & 6 & 5,90 & 38,40 \\
\hline Média & ral - & - & - & - & - & - & - & - & 32,51 \\
\hline
\end{tabular}

$\mathrm{Na}$ tabela 5, relacionam-se os valores genéticos aditivos preditos $(\hat{\mu}+\hat{a})$ e genotípicos $(\hat{\mu}+\hat{g})$, assumindo-se grau médio de dominância igual a $1 \mathrm{em}$ uma população com nível intermediário de melhoramento, ganhos genéticos e nova média da população dos 10 melhores genótipos da erva-mate aos 14 meses de idade para o caráter diâmetro.

Para o caráter diâmetro (Tabela 5) tais valores variaram de $21,31 \mathrm{~mm}$ a $23,50 \mathrm{~mm}$ e $20,75 \mathrm{~mm}$ a 22,33 $\mathrm{mm}$ respectivamente. Pôde-se observar que, de maneira geral, os valores genotípicos $(\hat{\mu}+\hat{g})$ preditos, foram superiores ao genético aditivo $(\hat{\mu}+\hat{a})$, tanto para altura como para diâmetro, podendo indicar maiores possibilidades de ganhos com o plantio de clones.
$\mathrm{Na}$ tabela 6, são apresentados os valores genéticos preditos para altura das plantas $(\mathrm{cm})$ por seleção com sobreposição de geração dos 20 melhores indivíduos de progênies de erva-mate, no município de Caarapó (MS).

Os dados obtidos remetem para a importância da estratégia a ser utilizada adotando-se pomares de sementes usando o modelo linear misto Reml/Blup, com as possibilidades seguintes: a) pomar testado de 1,5 geração com seleção parentais; b) pomares de sementes com seleção de indivíduos no experimento (pomares de segunda geração); c) Pomar com sobreposição de geração que é uma modalidade recente.

Tabela 5. Efeitos aditivos $(\hat{a})$, valores genéticos aditivos $(\hat{\mu}+\hat{a})$ preditos, efeitos genotípicos $(\hat{g})$ e valores genotípicos $(\hat{\mu}+\hat{g})$ para os 10 melhores genótipos de erva-mate aos 14 meses de idade, para o caráter diâmetro, em Caarapó (MS)

\begin{tabular}{|c|c|c|c|c|c|c|c|c|c|}
\hline \multirow[b]{2}{*}{ Bloco } & \multicolumn{2}{|c|}{ Propagação sexuada } & \multirow{2}{*}{$\hat{a}$} & \multirow{2}{*}{$\hat{\mu}+\hat{a}$} & \multirow[b]{2}{*}{ Bloco } & \multicolumn{2}{|c|}{ Propagação assexuada } & \multirow{2}{*}{$\hat{g}$} & \multirow{2}{*}{$\hat{\mu}+\hat{g}$} \\
\hline & Genótipo & Indivíduo & & & & Genótipo & Indivíduo & & \\
\hline 1 & 16 & 8 & 3,15 & 22,33 & 1 & 16 & 8 & 4,32 & 23,50 \\
\hline 1 & 22 & 8 & 2,58 & 21,76 & 1 & 22 & 8 & 3,86 & 23,04 \\
\hline 2 & 16 & 5 & 2,55 & 21,73 & 2 & 16 & 5 & 3,31 & 22,49 \\
\hline 1 & 16 & 1 & 2,02 & 21,20 & 3 & 20 & 6 & 2,57 & 21,75 \\
\hline 1 & 16 & 7 & 2,02 & 21,20 & 4 & 21 & 4 & 2,50 & 21,68 \\
\hline 1 & 16 & 2 & 1,79 & 20,97 & 1 & 16 & 1 & 2,43 & 21,61 \\
\hline 3 & 20 & 6 & 1,76 & 20,95 & 1 & 16 & 7 & 2,43 & 21,61 \\
\hline 4 & 21 & 4 & 1,76 & 20,94 & 4 & 13 & 9 & 2,34 & 21,52 \\
\hline 3 & 11 & 7 & 1,61 & 20,79 & 3 & 11 & 7 & 2,25 & 21,43 \\
\hline 1 & 16 & 5 & 1,57 & 20,75 & 4 & 21 & 5 & 2,13 & 21,31 \\
\hline Média & ral - & - & - & - & - & - & - & - & 19,18 \\
\hline
\end{tabular}


Tabela 6. Valores genéticos preditos para altura das plantas $(\mathrm{cm})$ por seleção com sobreposição de geração dos 20 melhores indivíduos de progênies de erva-mate, aos 14 meses de idade, em Caarapó, MS.

\begin{tabular}{|c|c|c|c|c|}
\hline Progênies & Indivíduos & Efeito genético aditivo predito (a) & Ganho Genético & Nova Média \\
\hline & & & & $\mathrm{cm}$ \\
\hline 5 & 1 & 8,5152 & 8,5152 & 41,0180 \\
\hline 20 & 8 & 6,1882 & 7,3517 & 39,8545 \\
\hline 20 & 7 & 5,7174 & 6,8062 & 39,3091 \\
\hline 20 & 0 & 5,5450 & 6,4909 & 38,9938 \\
\hline 21 & 7 & 4,8377 & 6,1603 & 38,6631 \\
\hline 20 & 8 & 4,7660 & 5,9279 & 38,4307 \\
\hline 20 & 6 & 4,6460 & 5,7453 & 38,2482 \\
\hline 16 & 9 & 4,6499 & 5,6069 & 38,1097 \\
\hline 20 & 9 & 4,6378 & 5,4698 & 37,9727 \\
\hline 9 & 10 & 4,3735 & 5,3582 & 37,8611 \\
\hline 9 & 6 & 4,3538 & 5,2636 & 37,7665 \\
\hline 16 & 7 & 4,3177 & 5,1845 & 37,6873 \\
\hline 16 & 0 & 4,3133 & 5,1119 & 37,6148 \\
\hline 21 & 8 & 4,2418 & 5,0486 & 37,5514 \\
\hline 16 & 5 & 4,2248 & 4,9907 & 37,4935 \\
\hline 20 & 2 & 4,1807 & 4,9286 & 37,4315 \\
\hline 4 & 4 & 3,9973 & 4,8684 & 37,3712 \\
\hline 4 & 0 & 3,9050 & 4,8067 & 37,3095 \\
\hline 21 & 6 & 3,7572 & 4,7438 & 37,2466 \\
\hline 4 & 9 & 3,6119 & 4,6868 & 37,1897 \\
\hline
\end{tabular}

Tabela 7. Valores genéticos preditos para diâmetro das plantas (mm) por seleção com sobreposição de geração dos 20 melhores indivíduos de progênies de erva-mate, aos 14 meses de idade, em Caarapó, MS.

\begin{tabular}{|c|c|c|c|c|}
\hline Progênies & Indivíduos & Efeito genético aditivo predito (a) & Ganho genético & Nova Média \\
\hline & & & & $\mathrm{mm}$ \\
\hline 16 & 8 & 3,1528 & 3,1528 & 22,3334 \\
\hline 16 & 0 & 2,8059 & 2,9794 & 22,1599 \\
\hline 22 & 8 & 2,5570 & 2,8453 & 22,0258 \\
\hline 16 & 5 & 2,5467 & 2,7706 & 21,9511 \\
\hline 16 & 1 & 2,0194 & 2,6204 & 21,8009 \\
\hline 16 & 7 & 2,0194 & 2,5202 & 21,7007 \\
\hline 20 & 2 & 1,7928 & 2,4163 & 21,5968 \\
\hline 21 & 6 & 1,7646 & 2,3348 & 21,5153 \\
\hline 11 & 4 & 1,7614 & 2,2711 & 21,4516 \\
\hline 16 & 7 & 1,6123 & 2,2052 & 21,3858 \\
\hline 16 & 5 & 1,5661 & 2,1471 & 21,3276 \\
\hline 13 & 9 & 1,5661 & 2,0987 & 21,2792 \\
\hline 21 & 9 & 1,5603 & 2,0573 & 21,2378 \\
\hline 21 & 5 & 1,5347 & 2,0200 & 21,2005 \\
\hline 21 & 3 & 1,4354 & 1,9810 & 21,1615 \\
\hline 20 & 7 & 1,3767 & 1,9432 & 21,1237 \\
\hline
\end{tabular}


Estes pomares são compostos de uma mistura de indivíduos selecionados no experimento com algum parental superior.

Nesse caso, somente um ranking simples por valores genéticos preditos é utilizado e os materiais superiores são incluídos no pomar não levando em consideração se pertencem à geração atual ou anterior (Resende, 2002a). Portanto, ao clonar os genitores selecionados, juntamente com os melhores indivíduos no teste de progênie, obtêm-se um pomar de sementes clonais misto.

Na tabela 7 são apresentados os valores preditos de diâmetro das plantas $(\mathrm{mm})$ por seleção com sobreposição de geração dos 20 melhores indivíduos de progênies de erva-mate.

Observa-se que entre os 20 melhores indivíduos selecionados para o caráter altura, a serem incluídos no pomar de sementes com sobreposição de geração, três deles, (identificados por "zero" na coluna "indivíduos") são os próprios genitores 20, 16 e 4 respectivamente. Desse modo, nota-se que, em geral indivíduos da geração atual tendem a ser melhores do que aqueles provenientes da geração anterior, como esperado. Entretanto, os parentais 20, 16 e 4 foram superiores a vários indivíduos da geração atual e deverão ser mantidos na população de melhoramento.

Por outro lado, para o caráter diâmetro das plantas (Tabela 7), entre os 20 melhores indivíduos selecionados para compor o pomar de sementes com sobreposição de geração, apenas um indivíduo (identificado por "zero" na coluna "indivíduos") é o próprio genitor 16 da geração anterior. É importante ressaltar, que o referido genitor possui potencial acima dos demais e deverá compor o pomar com sobreposição de geração.

\section{CONCLUSÕES}

1. A variabilidade genética demonstrada e as herdabilidades individuais e de progênies obtidas estimulam o monitoramento continuado das progênies e indivíduos no campo, com perspectivas de maximizar os ganhos genéticos na seqüência das avaliações.

2. Os valores genéticos preditos com propagação sexuada e assexuada indicam maiores possibilidades de ganhos com a implantação de plantios clonais, porém há que se considerar as estratégias a serem adotadas ao longo do programa de melhoramento da erva-mate em Mato Grosso do Sul.

3. A sobreposição de geração demonstrou que, em geral, os indivíduos da geração atual tendem a ser melhores do que aqueles provenientes da geração anterior. Deve-se, entretanto, considerar o genitor com potencial acima dos demais para a composição do pomar.

\section{AGRADECIMENTOS}

Os autores agradecem a CAPES e ao CNPq pelas bolsas concedidas e à FUNDECT pelo apoio financeiro ao projeto.

\section{REFERÊNCIAS}

ANDRADE, F.M. Diagnóstico da cadeia produtiva da ervamate (Ilex paraguariensis St. Hil.). São Mateus do Sul-PR: Consultoria, 1999. $92 \mathrm{p}$.

BOGUSZEWSKI, J. H. Uma história cultural da erva-mate: o alimento e suas representações. 2007. 130f.. Tese (Doutorado) - Universidade Federal do Paraná, Curitiba.

CORNELLIUS, J. Heretabilities and additive genetic coefficients of variation in forest trees. Canadian Journal of Forestry Research, Ottawa, v.24, p.371-379, 1994.

COSTA, R. B.; RESENDE, M. D. V. de; CONTINI, A. Z.; REGO, F. L. H.; ROA, R. A. R.; MARTINS, W, J. Avaliação genética dentro de progênies de erva-mate (Ilex paraguariensis St. Hil.), na região de Caarapó, MS, pelo procedimento REML/BLUP. Ciência Florestal, Santa Maria, v.15, p.371-376, 2005.

COSTA, R. B.; RESENDE, M. D. V. de; ARAÚJO, A. J.; GONÇALVES, P. de S.; HIGA, A. R. Selection and genetic gain in populations of Hevea brasiliensis with a mixed mating system. Genetics and Molecular Biology, v.23, p.671-679, 2000.

COSTA, R. B.; RESENDE, M. D. V.; GONÇALVES, P. S.; ARRUDA, E. J.;OLIVEIRA, L. C. S.; BORTOLETTO, N. Prediction of genotypic values for yield in rubber tree-clone test trials using REML/BLUP procedure. Crop Breeding and Applied Biotechnology, v.2, p. 575-582, 2002.

EHRLICH, P. R. The loss of diversity: causes and consequences. In: E.O. WILSON (Ed.), Biodiversity. Washington: Academy, 1988. p. 29-35.

FALCONER, D. S. Introdução à genética quantitativa. Viçosa: UFV, 1987. 279p.

FERREIRA, A.G.; KASPARY, R.; FERREIRA, H.B.; ROSA, L.M. Proporção de sexo e polinização em Ilex paraguariensis St. Hil. Brasil Florestal, p. 29-33, 1983.

GARCIA, C.H. Tabela para classificação do coeficiente de variação. Piracicaba: Instituto de Pesquisas e Estudos Florestais - IPEF, 1989. 10p. (Circular Técnica, 171).

GOVERNO DO PARANÁ. Parque Histórico do Mate. Disponível em: <http://www.pr.gov.br/phmate/ consumo.html>. Acesso em: $20 \mathrm{dez} .2007$. 
KAGEYAMA, P. Y.; VENCOVSKY, R. Variação genética em progênies de uma população de Eucalyptus grandis (Hill) Maiden. IPEF, p.9-26, 1983.

MISSIO, R. F.; SILVA, A. M.; DIAS, L. A. S.; MORAES, M. L. T.; RESENDE, M. D. V. Estimates of genetic parameters and prediction of additive genetic values in Pinus kesya progenies. Crop Breeding and Applied Biotechnology, v.5, p.394-401, 2005.

RESENDE, M. D. V. de. Genética biométrica e estatística no melhoramento de plantas perenes. Brasília: Embrapa Informação Tecnológica, 2002b. 975p.

RESENDE, M.D.V. Software Selegem-REML/BLUP. Colombo: Embrapa Florestas, 2002a. 67p.

RESENDE, M. D. V.; ARAÚJO, A. J.; SAMPAIO, P. T. B.; WIECHETECK, M. S. S. Acurácia seletiva, intervalos de confiança e variância de ganhos genéticos associados a 22 métodos de seleção em Pinus caribaea var. hondurensis. Revista Floresta, v.24, p.35-45, 1995.

RODIGHERI, H. R.; SCHLOSSNACHER NETO, L.; CICHACZEWSKI, I.F. Custos, produtividade e renda da ervamate cultivada na região de Guarapuava, PR. Colombo: EMBRAPA/CNPF, 1995. 22p. (Documento n. 24)

SAMPAIO, P. T. B. Variação genética entre procedências e progênies de Pinus oocarpa Schiede, Pinus caribaea var. hondurensis Barr. \& Golf. e Pinus maximinoi, H.E. Moore e métodos de seleção para melhoramento genético. 1996. 169p. Tese (Doutorado) - SEA/ Universidade Federal do Paraná, Curitiba.

SCHERER, R. A. Early selection of yerba mate (Ilex paraguariensis St. Hil.) in Argentina. Bonn: Rheinischen Friedrich-WilhelmsUniversitat, 1997. 58p.

SIMEÃO, R. M.; STURION, J. A.; RESENDE, M. D. V. Avaliação genética em erva-mate pelo procedimento BLUP individual multivariado sob interação genótipo $x$ ambiente. Pesquisa Agropecuária Brasileira, v.37, p.1589-1596, nov. 2002.

STURION, J.A. Variação genética de características de crescimento e da qualidade da madeira em progênies de Eucalyptus viminalis. 1993. 112f. Tese (Doutorado) Universidade Federal do Paraná, Curitiba:

STURION, J. A.; RESENDE, M. D. V. de. Programa de melhoramento genético da erva-mate no Centro Nacional de Pesquisa de Florestas da Embrapa. In: I CONGRESSO SUL AMERICANO DA ERVA-MATE. II REUNIÃO TÉCNICA DO CONE SUL SOBRE A CULTURA DA ERVA-MATE, 2., 1997, Curitiba. Anais... Colombo: Embrapa-Cnpf, 1997. 467p.

VAN VLECK, L. D.; POLLAK, E. J.; OLTENACU, E. A. B. Genetics for the animal sciences. New York :W.H. Freeman,1987. 391p.

VENCOVSKY, R.; BARRIGA, P. Genética biométrica no fitomelhoramento: Ribeirão Preto: Sociedade Brasileira de Genética, 1992. 496p. 